\title{
Die materiellen Voraussetzungen des geistigen Lebens in Byzanz - Handelskontakte mit Ostafrika, ihre Vorgeschichte und ihre Nachwirkungen
}

\author{
TILMAN KRISCHER
}

Das byzantinische Reich wird von den Historikern zumeist unter dem Gesichtspunkt der Kirchengeschichte betrachtet, während der Handel dabei kaum eine Rolle spielt. Aber das geistige Leben hat nun einmal auch seine materiellen Voraussetzungen: Ein gewisser Wohlstand ist erforderlich, damit die Bürger sich den Problemen in Ruhe widmen können. Der Wohlstand aber wird durch den Handel gefördert, und hier spielte viele Jahrhunderte hindurch das Gold eine wesentliche Rolle. In der kürzlich von Angeliki Laiou herausgegebenen umfangreichen byzantinischen Wirtschaftsgeschichte ${ }^{1}$ hat Klaus-Peter Matschke dem Thema Bergbau ein aufschlußreiches Kapitel gewidmet, ${ }^{2}$ in welchem er u.a. folgende Feststellung trifft: „In the seventh century, the Byzantine Empire lost a number of its most important mining regions to Arabs, Slavs, and other peoples. The political situation in the remaining parts of the empire was marked for some time by a high degree of instability, which must have greatly impended the orderly working of ore deposits and the establishment of costly installations for extracting metals."3 Diese Hinweise beziehen sich natürlich nicht nur auf die Goldgewinnung, sondern auf Erze aller Art, doch daß dabei dem Gold eine besondere Bedeutung zukommt, liegt auf der Hand. Bei den Wegen der Goldgewinnung aber geht es nicht nur um das, was nach den oben genannten Verlusten der Byzantiner entdeckt wurde, sondern auch um Wege, die bereits vorher bekannt waren und jetzt eine neue Bedeutung erhielten. Nur nebenbei sei erwähnt, daß Amerika entdeckt wurde bei dem Versuch, einen neuen Seeweg nach dem goldreichen Indien zu finden, und was man an Schätzen in dem neuen Erdteil vorfand, hat die kulturellen Verhältnisse in Europa maßgeblich beeinflußt. Also dürfte es kaum abwegig sein, wenn wir im Folgenden versuchen, einige nicht-byzantinische Quellen, den

1 A. E. Laiou (Hrsg.), The Economic History of Byzantium (DOS 39), Washington, D.C. 2002.

2 K.-P. Matschke, Mining, in: Laiou, The Economic History (s. Anm. 1), 115-120.

3 Matschke, Mining (s. Anm. 2), 117. 
Goldhandel und seine Folgen für das kulturelle Leben in Konstantinopel betreffend, zu erschließen.

Wir beginnen mit einem Aufsehen erregenden Fund, den der Afrika-Reisende Carl Mauch in den sechziger Jahren des 19. Jahrhunderts gemacht hat. Dabei stützen wir uns im wesentlichen auf die Darstellung, die Peter Hertel in dem traditionsreichen Perthes-Verlag herausgebracht hat. ${ }^{4}$ Die entscheidenden Punkte, um die es hier geht, sind zum einen eine aus örtlichem Granit errichtete Befestigungsanlage, wie sie anderwärts in Afrika nicht begegnet, und zum andern der Nachweis, daß man in dieser Region Gold gefördert hat. Mauch selbst vermutete, daß er das Goldland Ophir, von dem im Alten Testament die Rede ist, entdeckt habe. Doch diese Annahme hat sich inzwischen als fragwürdig erwiesen. Natürlich diente die Befestigungsanlage dem Schutz des geschürften Goldes, aber sie stammte gewiß nicht aus der Zeit von König Salomo. Den Angaben von Hertel zufolge $^{5}$ hat man in den 1950er Jahren der Bauanlage Holzproben entnommen, an denen in Chicago und London C14-Untersuchungen vorgenommen wurden, denen zufolge die Anlage aus der Zeit zwischen 500 und 700 n. Chr. stammt. Eben diese Epoche war eine Blütezeit des byzantinischen Reiches, und es könnte durchaus sein, daß von hier die Anregung kam zum Bau von steinernen Befestigungen. Diese Annahme aber setzt voraus, daß es zu jener Zeit einen Handelsverkehr zwischen Byzanz und Südafrika gab. Läßt sich das belegen?

In diese Richtung deuten Hinweise aus ganz anderen Quellen. Die Erkenntnisse nehmen von zwei Zeugnissen des Historikers Prokop ihren Ausgang. Dieser macht im zweiten Buch seiner Vandalenkriege (Kap. 14) folgende Bemerkung: „Und es begab sich, daß in diesem Jahr (535 n. Chr.) ein gar furchtbares Zeichen am Himmel erschien: Die Sonne, ohne Strahlkraft, leuchtete das ganze Jahr hindurch nur wie der Mond und machte den Eindruck, als ob sie ganz verfinstert sei. Außerdem war ihr Licht nicht rein und so wie gewöhnlich." ${ }^{\text {“6 }}$ Seinerzeit hat man ein solches Phänomen natürlich als ein böses Vorzeichen aufgefaßt, und so kam es, wie Prokop berichtet, zu allerlei Unruhen. ${ }^{7}$ Doch wie beurteilt man heute diese in der Tat höchst seltsame Erscheinung? Dazu hat der Archäologie-

4 P. Hertel, Zu den Ruinen von Simbabwe (Forschen und Entdecken), Gotha 2000.

5 Hertel, Zu den Ruinen von Simbabwe (s. Anm. 4), 148.

6 Procopii Caesariensis opera omnia, recognovit J. Haury, addenda et corrigenda adiecit G. Wirth,

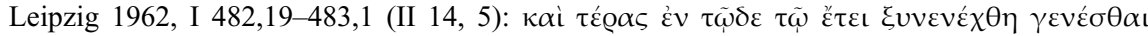

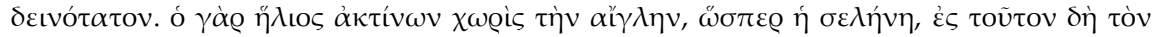

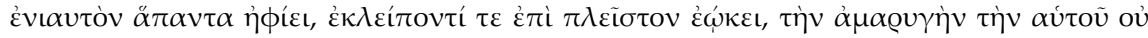

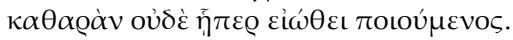

$7 \mathrm{Zu}$ diesem und dem nachfolgend beschriebenen Ereignis gibt es weitere byzantinische Quellen, insbesondere Cassiodorus berichtet ausführlich darüber. Eine aufschlußreiche Analyse bringt D. Stathakopoulos, Famine and Pestilence in the Roman and Early Byzantine Empire (Birmingham Byzantine and Ottoman Monographs 9), Aldershot 2004, 266-268. 
Korrespondent der BBC David Keys ein höchst aufschlußreiches Buch veröffentlicht. ${ }^{8}$ Gewisse Parallelen, auf die wir hier nicht einzugehen brauchen, legen es nahe, daß diese anhaltende Sonnenverfinsterung durch einen Vulkanausbruch ausgelöst wurde. Doch wo befand sich dieser, und mit welchen Mitteln läßt sich das Ereignis viele Jahrhunderte später beweisen? Hier helfen gewisse Errungenschaften der modernen Technik. Da jede Luftverschmutzung ihre Spuren in der Eisdecke des Nord- wie des Südpols hinterläßt, und ein gewisses Mindestmaß an Verschmutzung jedes Jahr auftritt, kann man anhand von Bohrkernen - ähnlich wie bei den Jahresringen der Bäume - sowohl das Datum einer gewissen Schicht ermitteln als auch die Stärke der Verschmutzung. Bei einer entsprechenden Untersuchung ergab sich tatsächlich, daß das Jahr $535 \mathrm{n}$. Chr. ein Zeitraum extremer Luftverschmutzung war. Die Spuren aber waren im Bereich des Nordpols und des Südpols etwa gleich stark, so daß der entsprechende Vulkanausbruch offenbar nicht weit vom Äquator entfernt stattgefunden hatte. Also machte man sich auf die Suche, und zwischen Java und Sumatra fand sich tatsächlich unterhalb des Meeresspiegels ein riesiger Krater. Dieser hatte bei seinem Ausbruch die beiden Inseln, die ursprünglich ein zusammenhängender Komplex waren, getrennt.

Doch was bedeutet dieser Vulkanausbruch im Zusammenhang unserer Untersuchung? Um hier weiterzukommen, müssen wir noch eine zweite Aussage des Prokop berücksichtigen. Im zweiten Buch seiner Darstellung der Perserkriege, Kap. $22,{ }^{9}$ berichtet er, daß sieben Jahre nach der Verfinsterung der Sonne in Konstantinopel die Pest ausbrach, eingeschleppt von Kaufleuten aus dem ägyptischen Pelusium. Später hat sich diese Seuche auch in anderen Teilen Europas ausgebreitet, und Prokop deutet sie, seinen Anschauungen gemäß, als Strafe Gottes. Doch die Wissenschaft unserer Zeit sieht auch in diesem Falle die Dinge anders. Die näheren Einzelheiten beschreibt David Keys in dem oben genannten Buch, dessen zweites Kapitel mit „Die Ursachen der Pest“ überschrieben ist. ${ }^{10}$ In groben Zügen ergibt sich folgendes Bild: Die Bakterien der Krankheit werden von Flöhen übertragen, die auf rattenähnlichen Wüstenmäusen leben, welche ihrerseits gegen die Krankheit immun sind. Normalerweise leben diese Tiere fern von den Menschen, doch bei anhaltender Dürre oder extremer Feuchtigkeit machen sie sich auf den Weg, und wo immer sie Menschen begegnen, wird die Katastrophe ausgelöst. Als die Heimat dieser Tiere nennt Keys eine Region namens Toniki in Ostafrika. Von hier können die Kaufleute die Küste entlang nach Norden fahren und gelangen sodann über den Golf von Aden und das Rote Meer in die Nähe von Pelusium. Kein Zweifel also, daß es im 6. Jahrhundert n. Chr. Handelskontakte

8 D. Keys, Als die Sonne erlosch - 535 n. Chr.: Eine Naturkatastrophe verändert die Welt, München 1999.

9 Haury/Wirth, Procopii Caesariensis opera omnia (s. Anm. 6), I 249-256 (II 22).

10 Keys, Als die Sonne erlosch (s. Anm. 8), 32ff. 
zwischen Ostafrika und Konstantinopel gab, und es spricht vieles dafür - nicht zuletzt die Schätze der Kirche -, daß dabei das Gold eine wesentliche Rolle spielte. $\mathrm{Da} ß$ diese Kontakte letztendlich bis hinunter nach Simbabwe reichten, erscheint möglich, läßt sich aber mit den bislang bekannten Mitteln schwerlich beweisen.

Doch da gibt es noch ein interessantes Zeugnis aus dem letzten Jahrzehnt des 15. Jahrhunderts, also einer Zeit, in der Konstantinopel bereits Sitz der Türken war. Ein Ritter namens Arnold von Harff hat damals eine Pilgerreise ins Heilige Land unternommen und dabei seine Erlebnisse sorgfältig aufgezeichnet. Die Handschriften wurden allerdings erst 1860 von E. von Groote ediert. ${ }^{11}$ Doch was hat eine Pilgerreise ins Heilige Land mit unserem Thema zu tun? Der Ritter reist zunächst von seiner Heimatstadt Köln nach Italien, wo er Rom und andere wichtige Städte besucht und seine Eindrücke schildert. In Venedig besteigt er sodann ein Schiff, um sich auf dem schnellsten Wege nach Alexandria bringen zu lassen.

Hier besichtigte er die Sehenswürdigkeiten der Stadt, und mit Hilfe von Kaufleuten gelingt es ihm, der Gebühr von 5 Dukaten, die ein Pilger normalerweise bezahlen muß, zu entgehen. Sodann begibt er sich nach Kairo und wird von den Palästen und anderen Bauwerken sehr beeindruckt. Nun schließt er eine vertragliche Vereinbarung ab mit gewissen Kameltreibern, die ihn über das Katharinenkloster zur Küste des Roten Meeres bringen. Hier angelangt, trifft er zwei genuesische Kaufleute, die in den indischen Ozean fahren und speziell Madagaskar aufsuchen wollen. Ihnen schließt er sich an. Zunächst wird der Weg an der Ostküste des Roten Meeres zu Lande zurückgelegt mit einem Zwischenaufenthalt in Mekka. Dann geht es weiter, abwechselnd zu Lande und zu Wasser, bis man nach etwa dreimonatiger Fahrt in Madagaskar anlangt. Dazu bemerkt der Autor wörtlich: „Item hie is gar eyn gude portz des mers, as alle koufflude van der werlt hie an landen yere koemenschaft zo drijven.“12 („Hier gibt es einen guten Meereshafen, in dem Kaufleute aus aller Welt landen, um ihren Handel zu treiben.") Kein Zweifel also, daß es zu dieser Zeit Handelsverbindungen zwischen Ostafrika und dem Mittelmeerraum (einschließlich Konstantinopel) gab. Über die Details des geschäftlichen Austauschs berichtet der Autor nicht, doch wir dürfen davon ausgehen, daß das Gold dabei eine nicht unwesentliche Rolle spielte. Konstantinopel aber war inzwischen seit mehr als einer Generation in türkischer Herrschaft. $\mathrm{Ob}$ sich die wirtschaftlichen Verhältnisse dadurch wesentlich verändert haben, werden wir später zu ermitteln versuchen.

Zunächst noch ein paar kurze Bemerkungen zur Heimreise des Ritters von Harff. In der Absicht, die Quellen des Nil zu entdecken, nimmt er seinen Weg

11 E. von Groote (Hrsg.), Die Pilgerfahrt des Ritters Arnold von Harff (Bewahrte Kultur), Hildesheim 2004.

12 Von Groote, Die Pilgerfahrt (s. Anm. 11), 145. 
über Äthiopien, fährt dann zu Schiff nilabwärts bis zu den Katarakten, dann geht es auf dem Landweg weiter nach Palästina, wo er alle wichtigen Stätten besichtigt. Seinen Rückweg in die Heimat nimmt er über Konstantinopel, wo er die Schlösser der Herrscher betrachtet und außerdem zahlreiche christliche Kirchen, die natürlich nicht mehr für den Gottesdienst verwendet, aber auch nicht abgerissen wurden. In einigen von ihnen hat man wilde Tiere gefangen gehalten. Von Konstantinopel aus gelangt der Ritter ins westliche Mittelmeer, umrundet Spanien, folgt der französischen Küste nach Norden, um dann auf dem Landweg über Paris seine Heimatstadt Köln wieder zu erreichen. - Soviel zu dieser Pilgerreise. Der Bericht macht deutlich, daß der Autor sich den christlichen Traditionen des Mittelalters verbunden fühlt; zugleich liefert er aber auch ein lebendiges Bild der geistigen Aktivitäten im Zeitalter der Entdeckungen und zeigt dabei, wie sehr diese Aktivitäten durch den allerorten blühenden Handel gefördert wurden.

In den nachfolgenden Jahrhunderten werden entsprechende Darstellungen des kulturellen Lebens im Nahen Osten vorwiegend von den Mitgliedern fürstlicher Gesandtschaften verfaßt. So reiste der Humanist Hans Dernschwam in den Jahren 1553-1555 mit einer Gesandtschaft Ferdinands I. von Wien zu Sultan Süleyman dem Prächtigen. Auf dieser Fahrt hat er ein Tagebuch geführt, das von Franz Babinger herausgegeben wurde. ${ }^{13}$ Einen umfassenden Überblick über den damit zusammenhängenden Materialbestand hat Wolfgang F. Reddig vorgelegt. ${ }^{14}$ Wie dieser Titel andeutet, stehen hier die religiösen Diskrepanzen im Vordergrund, ausgelöst durch die Belagerung Wiens von 1529. Was die kulturellen Leistungen anlangt, so beschreibt der Autor mit Vorliebe die Hinterlassenschaften der Antike. Doch darauf brauchen wir hier nicht näher einzugehen. Wir wollen uns statt dessen den Briefen von Lady Mary Montagu zuwenden, die im zweiten Jahrzehnt des 18. Jahrhunderts als Gattin des Gesandten der englischen Krone nach Istanbul fuhr und von den dortigen Verhältnissen überaus angetan war. Doch zuvor noch eine kurze Bemerkung von einem Reisenden des 17. Jahrhunderts. In den Jahren 1633-1639 reiste Adam Olearius mit einer Gesandtschaft des Herzogs von Holstein zum Zaren von Rußland und anschließend zum Schah von Persien. Von letzterem wurde er zum Abschied zu einem üppigen Mahl eingeladen, bei dem auch zahlreiche weitere Gäste zugegen waren. Und so bemerkt Olearius u.a.: „Desgleichen war ein reicher Jude erschienen, der zwischen Indien und Konstan-

13 F. Babinger, Hans Dernschwam's Tagebuch einer Reise nach Konstantinopel und Kleinasien (1553/55) (Studien zur Fugger-Geschichte 7), München 1923.

14 W. F. Reddig, Reise zum Erzfeind der Christenheit: Der Humanist Hans Dernschwam in der Türkei (1553-1555) (Weltbild und Kulturbegegnung 1), Pfaffenweiler 1990. 
tinopel handelte. ${ }^{\text {“15 }} \mathrm{Da}$ es in Istanbul auch eine jüdische Siedlung gab, ist bekannt, und hier wird deutlich, daß die von dort ausgehenden Handelskontakte offenbar sehr erfolgreich waren. Und entsprechend positiv sind die Urteile von Lady Mary Montagu zum wirtschaftlichen und kulturellen Leben der Stadt. Den Handelsverkehr betreffend, schreibt sie u.a. folgendes:

„Die Basare sind alle vortreffliche Gebäude, voll schöner Gänge, wovon die meisten auf Pfeilern ruhen. Sie werden sehr reinlich gehalten. Jedes Gewerbe hat seinen besonderen Gang, wo die Waren in der gleichen Ordnung wie in der New Exchange in London ausgelegt sind. Der Besisten oder Juweliergang zeigt so viele Reichtümer, eine solche Menge Diamanten und Edelsteine aller Art, daß sie das Auge blenden. Auch der Gang der Sticker ist sehr glänzend. Die Leute kommen hierher sowohl zur Belustigung als auch der Geschäfte wegen. Die Märkte sind meistens schöne Vierecke und vortrefflich mit Lebensmitteln versehen, vielleicht besser als in irgendeinem anderen Teile der Welt. “'16

Sollen wir wirklich davon ausgehen, daß die Verhältnisse in der christlichen Metropole der vorausgehenden Epoche wesentlich anders waren?

Noch eindrucksvolleren Luxus trifft die Autorin natürlich dort an, wo der Sultan und seine Angehörigen wohnen. Hier ein Beispiel:

„Gestern besah ich das Haus des verstorbenen Großwesirs, der bei Peterwardein fiel. Es war zur Aufnahme seiner königlichen Braut, der Tochter des jetzigen Sultans, gebaut, allein er erlebte es nicht, sie dort zu sehen ... Es liegt in einer der reizendsten Gegenden der Meerenge und hat einen schönen Wald am Abhang eines Hügels, der dahinter ansteigt. Es ist ungeheuer groß. Der Hüter versicherte mir, es wären achthundert Zimmer darin; ich will für diese Zahl nicht einstehen, weil ich die Räume nicht zählte, aber es sind gewiß sehr viele, und das Ganze ist verschwenderisch mit Marmor, Vergoldung und der auserlesenen Malerei von Blumen und Früchten verziert. Die Fenster sind alle aus feinstem Kristallglas, das aus England gebracht worden ist. Durchgehends herrscht die verschwenderischste Pracht, die man sich nur in einem Palast denken kann, den ein junger, üppiger Mann, dem der Reichtum eines großen Reiches zu Gebote steht, aufgebaut hat. Allein, kein Teil gefiel mir besser als die zu Bädern bestimmten Gemächer; es sind zwei ganz gleich gebaute, und man kann von einem in das andere gehen, die Becken, Brunnen und der Estrich alle aus weißem Marmor, die Decken vergoldet und die Wände mit japanischem Porzellan belegt. An diese stoßen zwei andere, deren erhöhter Teil ganz von einem Sofa eingenommen wird, in den vier Winkeln sind Wasserfälle, fast so hoch wie die Decke, sie fallen von Muschel zu Muschel aus weißem Marmor bis zu einem großen Becken am Boden und

15 D. Haberland (Hrsg.), Adam Olearius, Moskowitische und persische Reise: Die holsteinische Gesandtschaft beim Schah, 1633-1639 (Alte abenteuerliche Reiseberichte), Stuttgart/Wien 1986, 271.

16 M. Wortley Montagu, Briefe aus dem Orient, bearbeitet von I. Bühler (Bibliothek klassischer Reiseberichte), Frankfurt am Main 1982, 192. 
springen durch Röhren, mit denen es ringsum versehen ist, wieder so hoch wie die Decke. Die Wände sind eine Art Gitter, von außen mit Weinreben und Geißblatt bepflanzt, die eine Art grüner Tapete bilden und diesen reizenden Zimmern eine angenehme Dämmerung geben ... Der Garten entspricht völlig dem Hause. Lauben, Springbrunnen und Gänge liegen in angenehmer Regellosigkeit nebeneinander. Es fehlt nur die Zierde der Bildsäulen. Sie sehen also, mein Herr, daß diese Leute so rauh nicht sind, wie wir sie schildern. Es ist wahr, in der Pracht ist ihr Geschmack von dem unsrigen unterschieden, aber vielleicht besser. Fast bin ich der Meinung, daß sie einen richtigeren Begriff vom Leben haben. Sie verbringen es im Garten bei Musik, Wein und Leckerbissen, indes wir unser Gehirn mit politischen Entwürfen martern oder einer Wissenschaft nachgrübeln, die wir nie erfassen können, oder, wenn wir auch dahin gelangen, können wir die anderen nicht dazu überreden, denselben Wert darauf zu legen wie wir... Doch ich erlaube Ihnen, über die genießerische Erklärung zu lachen, die ich hiermit abgebe: Ich möchte lieber ein reicher Efendi mit all seiner Unwissenheit sein als Sir Isaak Newton mit all seiner Weisheit.“'17

So fragwürdig diese abschließende Bemerkung, die die Autorin unmittelbar vor ihrer Abreise von Istanbul formuliert hat, auch erscheinen mag, sie verdient insofern Beachtung als die neuzeitliche Wissenschaft Europas das Ziel verfolgt, beim menschlichen Handeln den Arbeits- und Materialaufwand zu verringern. ${ }^{18}$ Diese Ausrichtung hängt mit den Lebensverhältnissen im nördlichen Europa zusammen und nicht zuletzt mit einer Kälteperiode, die gegen Ende des 14. Jahrhunderts beginnt und in der Zeit des Dreißigjährigen Krieges ihren Höhepunkt erreicht. In dieser Epoche sind viele verarmte Bürger Englands in die Neue Welt ausgewandert, während in Konstantinopel bzw. Istanbul die Nöte offenbar geringer waren. ${ }^{19}$

17 Wortley Montagu, Briefe (s. Anm. 16), 206-208.

$18 \mathrm{Zu}$ diesem Thema vgl. u.a. F. de Gandt, Force and Geometry in Newtons Principia, Princeton 1995.

19 Einen guten Überblick über die geographischen und klimatischen Verhältnisse im byzantinischen Reich bringt J. Koder, Der Lebensraum der Byzantiner (Byzantinische Geschichtsschreiber Ergänzungsband 1), Wien 2001, vgl. u.a. 40ff. - Der Verf. dankt Michael Grünbart für wertvolle Hinweise zur Fachliteratur. 
